\title{
Politiques publiques de santé, logiques d' acteurs et ordre négocié au Burkina Faso
}

\section{Valéry Ridde}

\section{(2) OpenEdition}

1 Journals

Édition électronique

URL : https://journals.openedition.org/etudesafricaines/16603

DOI : 10.4000/etudesafricaines. 16603

ISSN : 1777-5353

Éditeur

Éditions de l'EHESS

\section{Édition imprimée}

Date de publication : 30 mars 2011

Pagination : 115-143

ISBN : 978-2-7132-2297-9

ISSN : 0008-0055

\section{Référence électronique}

Valéry Ridde, "Politiques publiques de santé, logiques d' acteurs et ordre négocié au Burkina Faso », Cahiers d'études africaines [En ligne], 201 | 2011, mis en ligne le 05 mai 2013, consulté le 22 avril 2022 URL : http://journals.openedition.org/etudesafricaines/16603 ; DOI : https://doi.org/10.4000/ etudesafricaines. 16603

Ce document a été généré automatiquement le 22 avril 2022.

(c) Cahiers d'Études africaines 


\title{
Politiques publiques de santé, logiques d' acteurs et ordre négocié au Burkina Faso
}

\author{
Valéry Ridde
}

1 Dans de nombreux pays à faible revenu, une myriade de politiques publiques de santé a été formulée ces dernières décennies dans le but équitable d' améliorer l' accès aux soins des plus pauvres. Les lecteurs, en tous les cas les plus anciens d' entre eux, se souviennent qu' ils avaient, à la fin des années 1970, le regard tourné vers Alma-Ata, la capitale actuelle du Kazakhstan. Dans cette ville de l' ex-Union Soviétique, la communauté internationale (134 pays) s' était réunie durant trois jours, sous l' égide de $l^{\prime}$ Organisation mondiale de la santé (oms) et du Fonds international de secours à l' enfance (Unicef). Une déclaration politique en faveur des soins de santé primaires était prononcée à cette occasion. Il s' agissait de rompre avec l' hospitalo-centrisme des systèmes de santé, et $\mathrm{d}^{\prime}$ orienter les réformes sanitaires vers $\mathrm{l}^{\prime}$ équité et une prise en charge globale et interdisciplinaire de la santé des communautés. La déclaration $\mathrm{d}^{\prime}$ Alma-Ata était en quelque sorte la première réforme sanitaire $d^{\prime}$ envergure internationale. Les gouvernements, en collaboration avec les communautés, devaient jouer un rôle essentiel pour diriger la réalisation des changements requis, car il s' agissait $\mathrm{d}^{\prime}$ une évolution majeure qui nécessitait une volonté intense pour lutter contre le statu quo (Collins 1994). Or le contexte de l' époque n' était pas le plus propice à l' intervention gouvernementale. $C^{\prime}$ est en effet durant cette période que des politiques dites d' ajustement structurel ont été imposées par la Banque Mondiale et le Fonds monétaire international (Nougtara et al. 2007). Le constat des conséquences de ces ajustements était dramatique, et la déliquescence des services publics (Olivier de Sardan 2000) s' opérait à l' aune de l' absence d' accès aux services de santé. Il fallait donc trouver de nouvelles solutions, particulièrement pour endiguer la réduction des moyens que l' État imposait, contre son gré, au système de soins. L' autofinancement des services de santé de la part des usagers est apparu alors comme une des réponses possibles (Deschamps 2000). La réflexion en faveur de l' autofinancement a grandement favorisé l' établissement d' une nouvelle réforme sanitaire : la politique de l' Initiative 
de Bamako (гв). Cette politique publique de santé a été formulée en 1987. Bien qu' elle ait été adoptée dans une capitale africaine, elle était largement $d$ ' origine exogène car elle a été entreprise sous l' impulsion des organisations internationales, soit l' oms et l' Unicef. À l' occasion de l' IB on s' est donné l' objectif d' améliorer la qualité des services par l' intermédiaire de l' autofinancement communautaire dans le but (efficace) d' augmenter l' utilisation des services de santé, tout en s' assurant que des mesures (équitables) soient prises pour que les indigents puissent avoir accès aux services. Or, force est de reconnaître que dans la majeure partie des pays africains, les plus pauvres sont toujours exclus des services de santé (Palmer et al. 2004 ; Ridde \& Girard 2004). Partout en Afrique, l' analyse des effets de l' Ів a montré que les acteurs responsables de sa mise en œuvre s' étaient essentiellement focalisés sur l' atteinte des critères relatifs à l' efficacité au détriment de ceux liés à l' équité (Gilson et al. 2000 ; Nitièma, Ridde \& Girard 2003 ; Ridde 2008). Ainsi, l' une des réussites sur le plan de l' efficacité de cette politique est $\mathrm{d}^{\prime}$ avoir contribué à rendre géographiquement accessibles les médicaments essentiels génériques et d' avoir accru, dans la plupart des cas, les taux d' utilisation des services de santé de première ligne. Cela étant dit, malgré les recommandations (et les inquiétudes) initiales en faveur de l' équité, les personnes qui sont dans l' incapacité de payer les soins demeurent exclues du système de santé, et aucune mesure en leur faveur n' a été mise en place.

Dans un article où il analyse, à Bamako, Dakar et Niamey, les stratégies des acteurs des systèmes de santé, Jaffré (1999) note que ces trois capitales ont en commun le fait que leur pays respectif a adhéré à l' IB mais qu' au-delà de la cohérence internationale de cette politique, ces pays ont fait des choix pragmatiques bien différents ayant été effectués diversement par les acteurs et les destinataires. Aussi, pour tenter de comprendre la focalisation des acteurs sur l' efficacité et non l' équité, il paraît utile de rappeler ce que disent certains experts ayant étudié la mise en œuvre des réformes et des politiques de santé. Plusieurs d' entre eux affirment que les échecs relatifs des politiques sanitaires trouvent leur origine dans le fait que la plupart $d$ ' entre elles se sont surtout centrées sur les recettes et les moyens de mise en œuvre; le contenu retenant plus l' attention que le processus (Brinkerhoff 1996 ; Walt \& Gilson 1994). Or, il semble déterminant de prendre en compte $d$ ' autres éléments fondamentaux, telles que les stratégies de mise en œuvre et les personnes-clés affectées, ce qui a suscité peu d' intérêt chez les chercheurs (Grindle \& Thomas 1991). Ainsi que le souligne Kingdon (1995), les politiques publiques peuvent $s^{\prime}$ expliquer par l' analyse de deux facteurs principaux, le processus et les participants à ce dernier. L' analyse de l' effet de la mise en œuvre de l' IB ne peut donc assurément faire l' économie de l' étude du jeu des acteurs. Par conséquent, à l' aide d' une étude de cas au Burkina Faso, la présente recherche tente de démontrer que la rencontre des acteurs sociaux peut en partie expliquer les effets de la mise en œuvre d' une politique de santé (IB). Mais avant cette démonstration empirique, il est nécessaire $d^{\prime}$ examiner les recherches anthropologiques qui sont $\mathrm{d}^{\prime}$ ores et déjà fécondes lorsqu' il s' agit de montrer l' importance du rôle des acteurs dans les politiques de santé.

\section{Le rôle des acteurs dans la mise en œuvre des politiques de santé}

3 À l' instar de nombreux auteurs (Rathwell 1998 ; Walt \& Gilson 1994), nous partons du principe que le rôle des acteurs est central dans la mise en œuvre et l' appropriation d' 
un changement. Il semble que cela soit d' autant plus le cas lorsqu' il s' agit d' analyser $l^{\prime}$ application d' une politique publique où des conflits liés aux valeurs peuvent amener les acteurs à tenter de bloquer ou d' entraver les réformes sanitaires (Gilson 1997). L' évolution des connaissances dans le domaine durant la dernière décennie nous a fait prendre conscience que le modèle classique du choix rationnel $n^{\prime}$ est plus le plus pertinent et qu' il faut reconnaître l' importance que jouent les acteurs sociaux dans la prise de décisions et la mise en œuvre des politiques (Brugha \& Varvasovszky 2000). En Afrique, des chercheurs viennent $d$ ' expliciter le pluralisme des normes, leur informalité et leur instabilité, obligeant les acteurs sociaux à de multiples ajustements permanents dans la gestion des affaires publiques et de l' action publique (Winter 2001). Autrement dit: "We view the process of policy change as one shaped significantly by the actions of individuals in strategic locations to influence a particular change" (Grindle \& Thomas 1991: 125). La conception de l' implantation d' une politique de santé peut être comprise diversement selon la perspective et les intentions des acteurs concernés (Walt 1994). De plus, en ce qui concerne l' IB, en associant les populations à la gestion locale des centres de santé et en accentuant le processus de décentralisation, la politique bouleverse complètement la distribution du pouvoir entre les acteurs locaux du système de santé ainsi qu' entre ces derniers et les acteurs centraux.

4 L' importance du rôle des acteurs dans la mise en œuvre spécifique de l' IB peut être illustrée par le cas de quelques pays. Au Burkina Faso, il a été allégué lors de la restitution des résultats $d^{\prime}$ une recherche sur les réformes de santé, que le ministère de la Santé a "fait cavalier seul » dans l' implantation des réformes. Les analystes vont même jusqu' à affirmer que cela explique, entre autres choses, l' absence dans les réformes de la prise en compte de l' accès aux soins pour les indigents (ABSP 2001). Ces constatations empiriques au Burkina Faso sont à mettre en relief avec ce qui, en 1992, figurait dans le document officiel de lancement de l' Initiative de Bamako. Il était souligné dans ce rapport que l' État $\mathrm{s}^{\prime}$ engageait à réaliser «des recherches opérationnelles sur l' indigence " (p. 26) et à prendre "des mesures pour la prise en charge des plus démunis » (Ministère de la Santé 1994 : 45). Plus de dix ans après, une étude a montré que les avancées en la matière avaient été très faibles en ce qui concerne le Burkina Faso (Ridde \& Girard 2004), comme au Bénin où l' efficience a pris le dessus sur l' éthique relative à la prise en compte des indigents (Ouendo et al. 2000). Au Mali, la viabilité des centres de santé a prévalu sur l' accessibilité (Maïga et al. 1999). La mise en œuvre de l' IB dans ce pays a été très difficile car l' administration était très minoritaire dans la volonté de développer cette initiative, et les professionnels de la santé n' ont pas hésité à manifester leurs réticences (Brunet-Jailly 1992). Dix ans après ces études maliennes, l' absence de démocratie au sein des associations de santé communautaire s' explique en zone urbaine par « des pratiques destinées à contourner des statuts faits pour assurer le caractère démocratique des élections" (Balique et al. 2001: 42) ou par un accaparement des associations par les oligarchies rurales. Cette constatation faite en 2001 n' est pas sans rappeler les inquiétudes émises à la fin des années 1980. Alors que le problème de l' accès aux soins des indigents était à peine effleuré, les premières expériences au Bénin et en Guinée avaient déjà organisé « des récompenses financières liées aux résultats » (p. 81) pour les agents de santé..., et sept ans après, les mêmes auteurs affirmaient que l' accès aux soins restait un problème irrésolu et qu' il faudrait "développer des mécanismes qui permettent d' assurer un accès équitable au système de santé» (Knippenberg et al. 1997 : 102). Les acteurs des 
réformes sanitaires en Afrique, comme dans bien d' autres continents, semblent plus préoccupés par la maximisation de leurs gains (Tizio \& Flori 1997), la thésaurisation des fonds communautaires (Ridde 2003) et la conservation de leurs pouvoirs (Beyer 1998) que par l' amélioration des systèmes de santé et la prise en charge des exclus permanents. Au Niger, la réduction de la participation des membres de la communauté à la seule acceptation de propositions préétablies pour la gestion de l' IB serait une explication aux résultats passables constatés après plusieurs années de soutien de la coopération hollandaise (Meuwissen 2002). Ces quelques exemples montrent combien la traduction et l' application concrète des politiques de santé sont influencées par les acteurs de leur implantation.

\section{Cadre conceptuel et stratégie de recherche}

5 Compte tenu de ce que nous venons d' évoquer à propos du rôle prépondérant des acteurs dans la mise en œuvre de l' IB, il paraît fondamental d' étudier finement la dynamique du jeu des acteurs. Aussi, il est intéressant d' avoir recours à des analyses en profondeur et de tenter de comprendre pourquoi de telles attitudes se manifestent et comment un programme $d^{\prime}$ origine exogène est "internalisé ", ou pas, et " réinterprété » par les acteurs sociaux (Long \& Long 1992). Pour cela, la présente étude $\mathrm{s}^{\prime}$ inspire des théories du changement social et $\mathrm{d}^{\prime}$ un des courants de la socioanthropologie du développement (Olivier de Sardan 2007). Car le cas étudié est bien celui de la mise en œuvre d' une politique de santé spécifique aux pays à faible revenu, d' origine largement exogène, où $l^{\prime}$ interaction entre les agents de développement (expatriés ou non) et les populations bénéficiaires est intense. Trois principaux courants se distinguent au sein de l' anthropologie du développement, et la présente étude s' inscrit dans le "néo-interactionniste»(Olivier de Sardan 2002). Selon ce dernier courant, il est important de faire l' inventaire des contraintes subies par les uns et les autres et de décrypter les stratégies déployées par ceux-ci. Les tenants de ce courant précisent, reprenant en partie ce que Strauss (1992:270) nomme «le potentiel créatif des individus », que les acteurs disposent d' une marge de manœuvre et qu' ils ne sont pas uniquement sous la dépendance de déterminants sociaux ou de pesanteurs culturelles. Le paradigme $d^{\prime}$ une certaine sociologie des organisations (Crozier \& Friedberg 1977) est très proche de ce courant de l' anthropologie du développement à tel point que certains de ses membres s' y réfèrent largement (Berche 1998). Dans le contexte du développement où, comme nous $l^{\prime}$ avons vu précédemment, les innovations sont souvent $d$ ' origine exogène, il est fondamental d' analyser le décalage entre les divers intérêts et rationalités des "développeurs" mais également des "développés ». Ainsi, il ne faut pas limiter notre analyse à la compréhension des groupes sociaux en tant que tels, mais il faut déplacer notre réflexion vers " $\mathrm{l}^{\prime}$ élucidation des liens $d^{\prime}$ échange, $d^{\prime}$ alliance, de rejet ou d' exclusion » (ibid.: 27) pour finalement tenter de comprendre comment les acteurs d' un projet de développement sanitaire en sont arrivés à cet ordre négocié ${ }^{1}$ (Strauss 1992) au détriment de l' équité d' accès aux soins de santé.

Dans cet article, pour saisir cet ordre négocié, il s' agit de mettre en exergue $l^{\prime}$ importance des logiques car "évaluer les politiques [...] c' est aussi identifier les logiques sociales, bureaucratiques et technocratiques, qui provoquent des décisions à responsabilité très limitée » dit Bernard Hours (2001 : 18). Olivier de Sardan (1995 : 126) nous explique que «au-delà d' une infinie variété potentielle des actions et réactions 
individuelles, on a affaire à un nombre relativement fini de comportements ». Il définit le terme de logique comme « les diverses lignes de cohérences que l' observateur déduit à partir $d^{\prime}$ une observation empirique $d^{\prime}$ ensembles de pratiques particulières différentielles, sans préjuger d' une théorie » (ibid.: 127). Dans les prochaines pages, nous allons ajouter des éléments empiriques aux logiques mises en évidence dans les travaux anthropologiques précédents (Jaffré \& Olivier de Sardan 2003; Olivier de Sardan 1990; Winter 2001), mais aussi, rendre compte empiriquement de nouvelles logiques rencontrées dans un cas précis au Burkina Faso.

7 Pour cela, une recherche ${ }^{2}$ a été menée dans un district sanitaire du Burkina Faso au moyen de la stratégie méthodologique de l' étude de cas (Yin 1994) et d' une enquête de terrain de type socio-anthropologique. Le cas étudié est un projet de coopération internationale d' une Organisation non gouvernementale (oNG) qui met en œuvre l' вв à travers un soutien aux services de l' État (une équipe cadre de district, ECD). Le cas a été sélectionné selon une démarche participative, avec les responsables du ministère de la Santé, en fonction de sa capacité à accroître notre compréhension du phénomène étudié (Stake 2000). Dans l' étude des politiques publiques (Lemieux 2002), on s' intéresse à quatre groupes stratégiques $d^{\prime}$ acteurs concernés en fonction de leur expertise (spécialisés ou non) et de leur place à l' égard de l' appareil gouvernemental (interne/externe). Ces groupes ont été définis pour l' IB et dans cette étude de cas :

8 - les intéressés (membre de l' ong et de comité de gestion, gérant de dépôt de médicaments) ;

9 - les particuliers (cultivateurs, indigents, utilisateurs (ou non) des services);

10 - les responsables (députés, consultants nationaux et internationaux, conseillers);

11 - les agents (infirmiers, médecins).

12 La recherche a utilisé de multiples sources de données: entrevues individuelles formelles et informelles (80), entrevues de groupe (4), étude de la documentation et des archives. De surcroit, puisque l' approche générale de la recherche nécessite une appréhension fine du contexte et des interactions individuelles et collectives entre ces quatre groupes $\mathrm{d}^{\prime}$ acteurs, il a été indispensable que nous fassions une longue immersion dans le milieu et que nous procédions à l' observation de ce dernier. Nous nous sommes ainsi inséré dans l' arène de notre recherche durant sept mois et avons participé aux interactions quotidiennes par de l' observation in situ en apportant une attention particulière aux relations entre les différents acteurs. Des notes ont été prises selon les démarches habituelles en anthropologie (Emerson et al. 1995). Plus de quarante situations où nous étions observateur non participant ont été analysées d' une manière formelle et méthodique. À cela, il convient évidemment $d^{\prime}$ ajouter les centaines $d$ ' interactions quotidiennes qui $\mathrm{n}^{\prime}$ ont pas fait l' objet de compte rendu mais qui ont indubitablement servi notre appréhension du contexte local.

\section{Le contexte social et sanitaire}

13 Le contexte de cette recherche est celui d' un district sanitaire administratif ${ }^{3}$. La population est très jeune, les villages sont de petite taille, la majeure partie des gens (de confession musulmane et faiblement scolarisés) sont des agriculteurs ou des éleveurs. Le contexte social est celui de la société des Mossi. Trois points fondamentaux (selon une représentation archétypale) se dégagent de l' organisation sociale des Mossi : 

sanitaires (Fs). Il est relativement dépourvu en personnel par rapport au reste de la région sanitaire et du pays. Neuf hommes forment l' équipe cadre de district (ECD) qui est chargée de la coordination des activités sanitaires, dont le responsable est le médecin-chef, présent depuis une dizaine d'années dans la région. Une ong implante un projet dans le district depuis 2001, à l' aide d' une équipe d' expatriés et de spécialistes nationaux. À cette époque, le district est très en retard dans la mise en place de l' IB, " C ' est pour cela que l' on nous a envoyés là-bas » (EI 12) ${ }^{4}$, nous dit un membre de l' oNG. En effet, d' après les informations obtenues auprès de l' oNG, en 1998, à la suite d' un besoin exprimé par la Direction régionale de la santé (DRS), et forte de son expérience ailleurs dans le pays depuis 1989 , l' ONG s' est proposée d'intervenir dans ce district. Il s' agit du cinquième projet de l' ong dans le pays. Pour des raisons liées au délai de traitement des dossiers de la part des bailleurs de fonds, les activités n' ont pu démarrer qu' en 2001, soit trois ans après la formulation du projet. Les objectifs du projet ont été déterminés en lien avec l' IB. Ainsi, l' objectif annoncé est d' améliorer $\mathrm{l}^{\prime}$ accessibilité géographique et financière aux médicaments essentiels génériques (MEG) et aux soins de santé primaires de la population de la région. Les principales activités consistent en des constructions de dépôts de MEG, des dotations en MEG et matériel médico-technique, des formations des comités de gestion (COGES), des gérants, des prescripteurs et des laborantins, un appui «institutionnel » à la DRS et au district (matériel, voyages d' études, etc.), des supervisions et contrôles des dépôt de MEG (DMEG), des séances de sensibilisation de la population. La mise en œuvre du projet a nécessité des ressources humaines propres (quatre expatriés, deux agents locaux, du personnel de soutien) mais aussi du personnel de l' État en poste dans la région sanitaire. Témoignage $d^{\prime}$ un fort roulement du personnel expatrié, sans compter le responsable du projet, ce ne sont pas moins de huit expatriés qui ont travaillé en trois ans pour la réalisation du projet.

\section{Quelques logiques d' acteurs}

$19 C^{\prime}$ est au moyen d' une fine observation d' une myriade d' interactions entre les acteurs sociaux concernés par la mise en œuvre de l' IB dans le district étudié, que nous avons été en mesure de repérer la récurrence de certaines logiques, concept défini préalablement, au sein de l' arène de cette recherche. Évidemment, les logiques, préalablement décrites dans les écrits anthropologiques, ont été prises en compte dans l' étude et ont constitué des points de départ. Cependant, $c^{\prime}$ est à l' aide d' observations empiriques que nous avons pu mettre en évidence la présence de dix logiques différentes. 


\section{L' accaparement}

20 La logique d' accaparement est comprise comme une stratégie qui vise à acquérir certaines ressources pour satisfaire des intérêts personnels ou corporatifs. Les ressources en jeu sont quasiment exclusivement des ressources financières. Néanmoins, ce qui nous restreint de qualifier cette logique d' accaparement financier, est que les données empiriques que nous avons recueillies ont " pointé du doigt » une stratégie $d^{\prime}$ accaparement $d^{\prime}$ un poste pour, in fine, acquérir des deniers supplémentaires. Autrement dit, ce que les acteurs recherchent par la mobilisation de cette logique est une mainmise sur l' argent, que ce soit $d^{\prime}$ une manière directe ou par l' intermédiaire $d^{\prime}$ un positionnement stratégique en occupant un poste favorable à une telle entreprise.

21 Le fait d' être membre d' un comité de gestion villageois est un parfait moyen d' avoir accès à ces ressources monétaires : « Il y a des gens qui se battent pour être dans le COGES, il faut bouffer » (EI 43). Il en est de même lorsque l' on est agent de santé et que l' on préconise et organise la gratuité des soins pour de tels fonctionnaires. Pour le reste, l' emprise directe sur l' argent s' effectue d' une manière, soit illégale (détournement : "Quelqu' un est venu un jour de Ouagadougou parce qu' on lui avait dit que les bâtiments qu' on avait construits étaient terminés, mais on $n^{\prime}$ en a pas » (FG 3) ; ventes de produits normalement gratuits «je rappelle que la vaccination est gratuite (Ob 16)»), soit profitant opportunément $d^{\prime}$ une situation légale ou réglementaire suffisamment floue pour qu' une telle stratégie soit envisageable. La manière d' utiliser les fonds générés par la vente des MEG et la tarification des actes $n^{\prime}$ a jamais été formellement précisée, favorisant ainsi l' usage inapproprié de cette manne. La kyrielle de séances de formation qui donnent lieu au paiement de per diem et la présence régulière de quasiment toujours les mêmes acteurs (médicaux), provoquant ainsi la mise à l' écart systématique de certains autres (administratifs), ont fait que l' on tente maintenant presque toujours $d^{\prime}$ imposer aux organisateurs/bailleurs de ces formations un poste d' administrateur de formation pour mieux " partager le magot ». Lors d' une réunion de cadres, nous avons constaté le fait que les participants reçoivent l' équivalent de deux jours de per diem alors $\mathrm{qu}^{\prime}$ elle ne dure qu' une journée, et un troisième per diem journalier est donné pour écouter une présentation d' une heure produite par les responsables d' une direction centrale (Ob 37).

\section{La neutra/domination}

Cette logique est en fait double, mais, compte tenu des données dont nous disposons et de l' imbrication des éléments empiriques, nous $n$ ' avons pas jugé utile de la séparer en deux. Pour la qualifier, nous sommes donc contraint d' user d' un néologisme illustrant le fait que cette stratégie consiste à employer sa fonction sociale ou administrative pour neutraliser ou dominer les autres. $L^{\prime}$ objectif de la mobilisation d' une telle logique par un acteur est donc $d^{\prime}$ exercer un certain pouvoir sur $d^{\prime}$ autres acteurs sociaux en vue de les dominer ou de réduire leurs capacités d'agir. En général, l' un ne va pas sans $l^{\prime}$ autre puisque, par exemple, lorsque l' on domine l' autre en ne lui fournissant pas d' information ou en la lui donnant $d^{\prime}$ une manière incompréhensible (cas de la formation), $c^{\prime}$ est aussi une manière de neutraliser sa capacité $d^{\prime}$ agir. 
23 L' étude des données empiriques paraît soutenir l' hypothèse que, dans une situation de hiérarchie sociale ou administrative entre les acteurs, la stratégie de la domination est adoptée. Cette constatation n' a évidemment rien d' extraordinaire puisque ce type de situation se rencontre très couramment, ici comme ailleurs. Cependant, dans la situation de la recherche, les éléments existants montrent qu' aucune autre option que celle de la logique de domination ne semble avoir été déployée lorsque l' on est en présence $d$ ' une telle relation pyramidale. Nous $n^{\prime}$ avons jamais rencontré de logique qui correspond à une confrontation directe. Il y a certes des débats et par moment même l' instauration de blocages, mais ceci n' est jamais scénarisé selon une démarche directe, où les acteurs auraient à interagir instantanément. On usera plutôt d' une stratégie $d$ ' évitement; nous reviendrons sur ce sujet plus loin. Cette relation dominatrice existe entre les gens du Nord et ceux du Sud, entre les développeurs et les développés mais aussi entre les habitants du Burkina, dans la mesure où un gradient social définit le rapport entre ces acteurs locaux. Cette relation sociale est bien souvent aussi la résultante d' une relation administrative (médecin/infirmier, burkinabé/ expatrié) tels que les exemples suivants l' explicitent. Des employés burkinabè de l' onG, en conflit avec un expatrié, $n$ ' ont $d^{\prime}$ autre choix que $d^{\prime}$ attendre le départ de celui-ci pour espérer être mieux considérés dans l' organisation. Lors $d$ ' une réunion, le

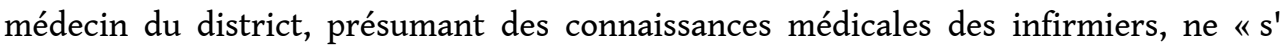
abaisse " pas à leur fournir une explication pédagogique sur un élément que ces derniers ne maîtrisent apparemment pas. Dans un village, un membre de l' ECD en visite de supervision réagit de la même manière avec un infirmier chef de poste (ICP) et, telle une boule de neige sociale, un ICP tout autant avec un villageois responsable communautaire ou encore un malade. La langue française est parfois utilisée au cœur de cette logique. Par exemple, on demande à des membres de COGES, des paysans issus des villages, de se présenter en français, lors d' une réunion, alors qu' ils ne semblent pas comprendre cette langue. Puis, lorsque vient leur tour de parler, ils ne savent pas pourquoi ils doivent prendre la parole et interrogent le seul collègue qui semble avoir compris la requête. Sur un autre registre de domination, les soignants $n^{\prime}$ acceptent pas les reproches ou les questions que posent des soignés. Lors d' une visite, alors qu' un père paraît se plaindre du traitement apporté à son enfant dans un dispensaire, l' infirmière dit «Nous ne sommes pas allés les chercher [...] ce n' est pas lui qui va me dire ce que je dois faire " (V 10). Tout porte donc à croire que les relations humaines sociales, évoluant dans un contexte de forte stratification sociale, s' organisent de telle sorte que la domination des uns sur les autres perdure et que la contestation ne soit ni envisageable ni envisagée.

Cependant, à partir du moment où le lien n' est plus unilatéral mais conjoint, ce n' est plus une stratégie de domination qui l' emporte mais bien, dans certains cas, une volonté de neutralisation qui, bien souvent, est associée à un but utilitariste. Par exemple, un médecin donne un per diem de 5000 FCFA à un agent pour sa participation à une réunion, alors que, $d^{\prime}$ une part, ce dernier est arrivé très en retard, et, $d$ ' autre part, le médecin n' est pas obligé de le donner (Ob 37). Dans un autre cas, une relation de pouvoir s' instaure entre un expatrié de l' ONG et un Burkinabé à travers la distribution de per diem. Puisqu' ils $\mathrm{n}^{\prime}$ ont aucune relation hiérarchique, ils vont user de cette ressource financière pour se neutraliser, animés par la volonté de poursuivre la collaboration indispensable à la mise en œuvre du projet qui ne peut s' arrêter et au maintien de l' accès aux incitatifs financiers. $C^{\prime}$ est le cas, par exemple, lorsque l' oNG 
" court ", pour reprendre les mots entendus, après un agent ( $\mathrm{Ob} 42$ ), pour lui remettre le per diem qu' il a refusé afin d' être en mesure de continuer à travailler avec lui, sa participation étant indispensable à la réussite, du moins à la réalisation, des activités relatives au projet. De même, le médecin expatrié, qui se trouve être le seul à disposer du même capital social que le médecin du district, $n$ ' est pas vu d' un bon œil et perçu par ses collègues médecins des autres districts de la région comme un élément perturbateur (EI 29, EF 11). À l' inverse, les expatriés pharmaciens qui n' ont pas d' alter ego professionnel dans le district de santé ne font absolument pas face aux mêmes difficultés relationnelles.

\section{Le discours}

La troisième logique observée est celle de la construction d' un discours. Les émetteurs de ce discours $s^{\prime}$ organisent pour développer une argumentation, essentiellement destinée aux étrangers, dans le but de fournir des informations qui ne sont qu' une façade de la situation locale.

Cette manière de discourir provient essentiellement des agents de santé et des membres de la communauté. Les récepteurs de ces discours de façade sont les bailleurs de fonds ou les intervenants de l' aide internationale ainsi que les enquêteurs qui viennent tenter de comprendre la société locale en interrogeant les personnes concernées. Cependant, user d' un discours lénifiant n' est pas l' apanage des seuls développés à $\mathrm{l}^{\prime}$ intention des développeurs, comme on aurait tendance à le croire. Ces derniers, des agents de santé par exemple, emploient également une telle stratégie. Lorsque l' on étudie les informations existantes dans une perspective téléologique, il nous semble avoir décelé trois finalités principales du déploiement $d^{\prime}$ une telle stratégie.

La première finalité consiste à faire croire que la société locale est une société juste qui se préoccupe des plus pauvres. On cherche notamment à nous expliquer que plusieurs actions ont été mises en branle pour améliorer l' accès aux soins des indigents, qu' un processus de planification allant dans ce sens est en marche : «On dit aux districts d' inclure ce volet dans leur plan d' action [...] non ils ne l' oublient pas, ils le font » (EF 10). On va, par exemple, dissimuler à l' étranger qui $s^{\prime}$ interroge sur le prix des accouchements dans l' objectif de comprendre le niveau d'accessibilité financière le fait que le montant donné ( $500 \mathrm{~F}$ ) ne prend pas en compte l' ensemble des dépenses nécessaires à un tel acte médical (5 $000 \mathrm{~F}, \mathrm{~V}$ 10). On va affirmer que les directives centrales pour plus d' équité sont respectées alors qu' elles ne le sont pas. La seconde finalité concerne la volonté de présenter une société stable, sans conflit, égalitaire et animée par un profond désir de paix sociale. On va ainsi, par exemple, s' ingénier à occulter l' existence de certains conflits dans un document officiel de planification quinquennal, destiné à circuler largement : «Le mauvais climat social dont vous avez parlé, est-ce que $c^{\prime}$ est encore le cas ? Est-ce que $c^{\prime}$ est bien de mettre cela dans un document officiel qui va traîner? » (Ob 38). Enfin, dans un contexte évident de rareté de ressources, il faut user d' un discours qui sera en mesure, non seulement d' attirer l' attention sur la misère du monde et les besoins locaux, mais aussi de limiter la mise au jour de problèmes insurmontables par l' attribution $\mathrm{d}^{\prime}$ une aide financière, sous la forme $d^{\prime}$ un projet de coopération internationale par exemple. L' un des premiers réflexes des paysans est de dire qu' ils sont tous pauvres - un ancien responsable au 
siège occidental de l' ONG nous disait également que les indigents $n$ ' existent pas dans cette région du pays - et qu' ils ont besoin d' aide : « Tout le monde est pauvre, nous $\mathrm{n}^{\prime}$ avons rien, nous sommes tous indigents » (FG 1).

\section{Le clientélisme}

La rencontre de la logique du clientélisme ne s' explique pas uniquement par la volonté des acteurs de développer des pratiques de corruption, par exemple. Ici, cette stratégie est plutôt comprise au sens large du terme, $c^{\prime}$ est-à-dire simplement $l^{\prime}$ usage et $l^{\prime}$ entretien de ses relations personnelles à des fins diverses. En outre, notre présence sur le terrain nous a permis d' entendre les acteurs raconter que l' utilisation d' une telle logique pouvait avoir été efficace, mais que cela n' avait pas toujours été le cas.

Lorsque l' on s' attarde sur les réussites, autrement dit les stratégies de clientélisme qui ont porté leurs fruits, on découvre que certaines pratiques ont été bénéfiques à certains individus, mais au détriment de la collectivité, tandis que $\mathrm{d}^{\prime}$ autres ont été collectivement utiles. Le premier cas, bien connu, se constate notamment lorsqu' un particulier fait appel à ses connaissances (agents de santé) pour avoir accès aux soins ou à certains médicaments normalement gratuits : «Quand tu as ton parent au centre de santé, ils t' aident beaucoup » (FG 1). Quand un homme politique, ancien ministre de surcroît, emploie ses relations pour disposer $d^{\prime}$ un financement permettant de construire un dispensaire dans son village d' origine alors que cela n' est pas prévu dans la carte sanitaire, $c^{\prime}$ est encore le cas. Les conséquences (ou les causes ?) de cette implantation peu rationnelle ( $\mathrm{d}^{\prime}$ un point de vue de santé publique) ajoutées aux "querelles intestines interethniques " (vs 6) consistent en l' absence de consultations et en un dispensaire vide lors de nos visites. En revanche, la pratique du clientélisme au profit de la collectivité est à noter dans l' utilisation des fonds issus des Journées nationales de vaccinations (JNV) pour entretenir les relations avec les agents de santé communautaires (ASC) ne disposant que de cette occasion pour obtenir quelques subsides. Certes, il s' agit de bénéfices pécuniaires personnels, mais puisque ces ASC sont censés fournir quelques services de base aux populations locales (dont l' efficacité reste à démontrer), nous pouvons avancer que cette pratique clientéliste sert la collectivité, du moins à moyen terme. Un autre exemple collectivement favorable est à trouver dans la technique employée par un responsable d' hôpital, qui profite de ses relations politiques pour qu' un agent du ministère de l' Action sociale soit nommé et ouvre un service social destiné à favoriser l' accès aux soins des indigents.

À deux reprises, nous avons été témoin d' une tentative d' application de cette logique, sans que, pour autant, la personne qui l' employait ait atteint son but. Par exemple, nous avons observé une tentative échouée de la part d' une personne apparemment nantie pour obtenir un certificat de décès (qui donne droit à une pension de l' État pour la famille du défunt) auprès d' une agente de santé, qui n' avait pas vu le corps (V 10). Ainsi, la pratique de la logique de clientélisme n' est pas toujours couronnée de succès (impossibilité $d$ ' obtenir une affectation après avoir intercédé auprès du secrétaire général... " donc on va voir le ministre » (EI 46)), même si la prévalence des réussites semble indéniablement importante. 


\section{L'opacité}

31 La logique de l' opacité se définit comme la volonté délibérée de certains acteurs de cacher des informations à d' autres protagonistes.

32 Le processus par lequel cette occultation s' opère est double, mais aboutit à la même conséquence : la tenue à $\mathrm{l}^{\prime}$ écart de certains acteurs sociaux. Soit l' information n' est tout simplement pas fournie aux personnes concernées, comme, par exemple, l' information sur la manière dont les fonds générés par le recouvrement des coûts sont utilisés (« Tu ne sais pas ce qu' ils font avec tout cet argent » [FG 2]), ou encore, lors de la présentation des conclusions des procédures de contrôles des MEG en l' absence des membres du comité de gestion formé par les habitants du village; soit $c^{\prime}$ est $l^{\prime}$ accès même aux informations que l' on rend impossible, en gardant, par exemple, tous les rapports dans une seule armoire dont une seule personne a la clé (Ob 43) ou encore en enfermant les registres de la consultation médicale dans le bureau et en emportant la clé lorsque l' on quitte les lieux : "Je vais voir s' il a laissé cela sur sa table, dans le cas contraire, cela sera difficile » (EI 46).

\section{La connivence}

33 La stratégie de la connivence est très proche de celle du clientélisme, mais elle s' en différencie doublement dans le cas précis de cette étude. D' une part, elle se déploie uniquement, dans le cas de ce district, au sein de la catégorie des agents de santé, quelle que soit leur position dans l' échelle hiérarchique du système de santé ; d' autre part, en forçant un peu le trait, elle se rapproche de pratiques mafieuses ou, plus joliment dit, de cliques ou encore d' ententes tacites. Cette logique se définit par le fait que les agents de santé se couvrent les uns les autres; en dépit de certaines malversations ou pratiques peu éthiques, ils décident de ne pas dénoncer leurs collègues, ou ne veulent tout simplement pas prendre de risque en les dénonçant par peur d' être exclus du réseau et, in fine, de ne plus bénéficier des largesses de ce dernier.

Les superviseurs du district découvrent des pratiques frauduleuses (double facture, dépenses somptuaires) de la part de leurs collègues qui sont en fonction dans un dispensaire, mais ne disent rien (vs 1). D' autres superviseurs ne prennent pas une position très ferme sur la qualité des soins prodigués aux patients, surtout « si tu viens à midi et que le major [1' infirmier] a faim » (Ob 1). Car, dans un contexte de rareté de ressources et d' occasions de disposer de revenus supplémentaires par l' intermédiaire de per diem ou d' utilisation abusive de certaines sommes, il s' agit de ne pas s' exclure $d u$ système. La feuille de présence des superviseurs lors d' une visite d' un dispensaire reculé, donnant lieu à des per diem relatifs à un projet national, est signée par l' infirmier responsable du dispensaire supervisé. Il confirme ainsi la présence de toutes les personnes dont le nom a été inscrit sur le formulaire, alors même qu' une partie est absente (vs 3). Certes, de temps à autre, la hiérarchie sanitaire $s^{\prime}$ étonne de ces pratiques, dit quelques mots pour préciser leur rôle néfaste mais, revenant à la logique du discours, ces étonnements sont essentiellement virtuels et perçus comme tels par les acteurs du système. Lors $\mathrm{d}^{\prime}$ une réunion, le responsable explique à tous les infirmiers du district que « l' an passé, il y a eu des problèmes de répartition des ressources [...] il faut faire attention, $d^{\prime}$ autant $q u^{\prime}$ avec le cas de PFA [enfant suspecté de polio], on est dans le collimateur de l' oms, [...] donc ne soyez pas trop gourmand » (Ob 16). Il arrive 
aussi que ces responsables constatent de telles malversations, mais ils ne disent rien et éludent le problème, et ensuite, ce qui va tout de même beaucoup plus loin, formulent quelques conseils pour que cela ne soit pas trop visible. $C^{\prime}$ est notamment ce qui se passe, $\mathrm{d}$ ' une part, lorsque l' on n' interdit pas les médicaments de spécialités dans le dépôt de MEG alors que leur présence est interdite mais plutôt que l' on conseille de les cacher : «Si vous voulez vous mettez cela ailleurs, mais pas dans le dépôt » (vs 2), et, d' autre part, lorsqu' un superviseur explique aux infirmiers la manière de camoufler dans les comptes la distribution de jetons de présence aux membres des COGES, « Il y a lieu de changer d' intitulé [dans la comptabilité], notez "repas communautaires" ” (vs 7). Contrairement au détournement collectif des per diem qui ne trouve aucune justification autre que l' enrichissement personnel, ces deux derniers exemples sont $\mathrm{l}^{\prime}$ illustration de la difficulté des agents à respecter certaines règles alors que tous les besoins ne sont pas couverts, comme, par exemple, en ce qui concerne ces médicaments de spécialités ayant certainement été donnés gratuitement aux dépôts par une association $d^{\prime}$ un pays riche et pouvant servir à soigner gratuitement certains malades... ou arrondir les fins de mois d' agents de santé peu scrupuleux.

\section{L' évitement}

35 La stratégie ici décrite est qualifiée de logique d' évitement dans la mesure où elle fait référence à des comportements humains qui cherchent à tout prix à éviter la survenue $\mathrm{d}^{\prime}$ un conflit direct entre les acteurs sociaux.

Trois buts principaux sont visés par la mise en œuvre d' une telle logique. D' abord, comme nous l' avons vu à plusieurs reprises, il semble bien que les acteurs recherchent l' instauration et la pérennité de la paix sociale. Il ne faut pas créer de troubles, ce qui implique certaines fois de passer sous silence $-c^{\prime}$ est le prix à payer pour que le calme règne - des malversations constatées ou des erreurs commises. Ensuite, éviter les conflits est également un moyen d' avancer dans la mise en œuvre du projet, par exemple. Ne pas s' attarder sur certains problèmes permet de poursuivre les travaux et d' aller de l' avant, ce qui a pour effet pervers de laisser en suspens des difficultés. Enfin, le dernier objectif de telles pratiques est de se protéger contre d' éventuelles poursuites, judiciaires notamment. Puisque l' on n' a pas réglé ou cherché à régler les problèmes de l' accessibilité financière des indigents, on demande au patient, contraint de quitter le centre de santé sans avoir obtenu les traitements faute de moyens financiers, de signer une décharge pour que l' agent de santé soit protégé. On évite ainsi de trouver une solution à ce problème et, $d$ ' une pierre deux coups, on se protège contre une hypothétique plainte du malade ; hypothétique, car le contexte local ne se prête évidemment pas à de tels procès. Cette pratique pourrait traduire l' influence nord-américaine (et maintenant européenne) : après la privatisation voici le temps de la « judiciarisation » du secteur de la santé.

Pour atteindre ces objectifs à l' aide de la logique d' évitement, il nous semble avoir décelé la mise en route de deux processus particuliers. Le premier est la recherche du transfert de la responsabilité selon une chaîne, une cascade, allant des bailleurs de fonds, à l' ONG, aux agents de santé et aux particuliers. Les agents font signer une décharge aux patients. Pour justifier leur pointillisme dans les contrôles, les membres de l' ong renvoient la balle dans le camp des bailleurs de fonds en affirmant qu' ils font ces travaux à leur demande : " Nous, on regarde les registres pour nos statistiques, on a 
besoin de cela, ce n' est pas prévu dans le contrôle, c' est les bailleurs de fonds » (V 3). Ainsi, personne ne souhaite prendre de responsabilité, ce qui est évidemment le meilleur moyen d' éviter des conflits mais aussi les pratiques de reddition de comptes. Cela peut notamment s' expliquer par le fait que « la responsabilité, on sait d' où cela vient, mais personne ne vient prendre la décision » (Ob 3). La seconde manière de faire est de garder le silence, d' éviter la critique et finalement de tenter de faire plaisir à tout le monde en même temps. La plupart du temps lors des réunions, les acteurs ne font pas usage d' un jugement critique de ce qui se dit ou de ce qui est écrit. Lors d' un atelier de validation $d^{\prime}$ un projet d' établissement, de nombreuses remarques sont émises pour retirer ou reformuler des phrases qui démontrent des problèmes internes, «ils ne veulent pas se poignarder », nous dira notre voisin de table (Ob 41), «le seul problème c' est qu' ils n' extériorisent pas leurs problèmes » (vs 3), entendra-t-on ailleurs. Pour éviter les conflits avec les responsables d' une formation sanitaire et pour "se partager le gâteau ", le médecin-chef décide de demander que deux personnes de ce dispensaire, alors qu' il n' en était prévu qu' une seule, fasse des vaccinations (Ob 44). Une autre fois, il tente $d^{\prime}$ 'organiser un vote, car il ne parvient pas à imposer son leadership à propos de la manière de marquer les maisons après le passage des vaccinateurs. Mais les résultats ne sont pas satisfaisants, et il organise un second vote. De nouveau, les résultats ne contentent personne, et il décide finalement de laisser libre court aux initiatives personnelles pour le marquage, sans qu' une décision commune ne soit prise (Ob 44). Les membres de l' ONG « achètent » le silence des agents de santé en leur donnant des per diem pour que ces derniers ne troublent pas la fin du projet et la présentation de son évaluation. Le responsable de l' ong décide ainsi d' accorder un haut (et inhabituel) taux de per diem aux participants à la restitution des résultats pour « avoir la paix » (Ob 45), tout comme nous aurons compris que l' « on ne va pas dire au MCD que ce n' est pas bien, sinon il va tout bloquer et on ne pourra pas avancer $»(\mathrm{Ob} 46)$.

\section{La suspicion}

La mise en œuvre de cette logique se rencontre dans des processus relationnels qui peuvent se définir comme la suspicion, a priori, de mauvais comportements ou d' incapacité de l' Autre. Le corollaire évident de cette stratégie est le manque de confiance qui règne entre les acteurs sociaux concernés par ces relations interpersonnelles. Cela ne veut évidemment pas dire que dans $l^{\prime}$ arène de notre recherche, personne ne fait confiance à personne, mais nous avons, malgré tout, rencontré un certain nombre de situations, récurrentes et cohérentes, proches de cette logique. Ce qu' il faut d' emblée noter est que, contrairement à ce que nous pourrions penser, cette situation n' est absolument pas propre aux relations qu' entretiennent les expatriés (du Nord) avec les Burkinabè (du Sud). De nombreuses situations ont montré que cette logique ne s' est pas cantonnée à ces liens particuliers. Les rapports existants entre les paysans membres $d^{\prime}$ un coges et le reste du village, de même que ceux entre les membres de l' ONG et le personnel de santé ou encore ceux entre certains agents de santé eux-mêmes sont également, dans nos constats empiriques, parfois empreints d' une logique de suspicion.

On ne s' étonnera pas de constater que l' objet central de cette suspicion est l' argent. Certains vont penser que $d^{\prime}$ autres ne sont intéressés que par $l^{\prime}$ argent ou, tout 
simplement, qu' ils organisent des malversations, ou qu' ils détournent allégrement des fonds. Ce sentiment émane du discours des acteurs relevant tant de l' ong que des services de santé, il n' est l' attribut ni des premiers ni des derniers. Un expatrié dit: "Si on vous prévient [pour les contrôles financiers], vous avez le temps de trafiquer les chiffres » (V 10), tandis qu' un agent de santé précisait : "Nous on est pressé que l' ONG $\mathrm{s}^{\prime}$ arrête, $\mathrm{c}^{\prime}$ est la gendarmerie [...] on n' est pas des voleurs " (V 3). À propos des questions financières, les agents reprochent à l' oNG qu' « ils ne sont pas si pauvres que cela pour nous donner des copies » (Ob 32) et qu' « ils disent qu' ils ont de l' argent mais ils ne déboursent rien » (Ob 34). La conséquence est que les acteurs ont peur de voir de nombreuses sommes $d$ ' argent disparaître dans la nature ou dans les poches des autres. L' effet peut aussi être désastreux pour l' accès aux soins. Préoccupés par la politique du recouvrement des coûts et par l' obligation de faire payer les patientes, des agents décident, suspectant certaines femmes bénéficiaires de crédits - octroyés le jour où elles n' étaient pas en mesure de payer -, de conserver leur carnet de santé en guise de garantie (V 2). Ce type de pratique ne favorise ni l' utilisation des services ni la confiance entre les usagers et les soignants. Un autre point intéressant doit être ajouté, dans le contexte du présent chapitre. L' amoncellement d' argent que provoquent la mise en œuvre de l' IB et le paiement des médicaments fait naître, au cœur de cette logique de suspicion, la peur des membres des COGES d' utiliser cet argent: « Il y a la peur de dépenser, peur que l' on va dire qu' ils ont bouffé » (EI 19). Les données que nous avons collectées $\mathrm{n}$ ' ont pas permis d' inférer sur le fait qu' ils auraient eu l' idée de l' employer pour améliorer l' accès aux soins des indigents, ce dont nous doutons cependant. Mais cet élément est éclairant car il montre que, dans certains contextes sociaux (locaux ?), le fait même de dépenser de l' argent provenant de la communauté est une gageure qui demeure, en partie, socialement contingente.

Mais la suspicion ne concerne pas que des questions monétaires. Alors qu' un formateur burkinabé demande à un expatrié de l' ong la possibilité, en début de journée de formation, de distribuer le programme aux participants, ce dernier $s^{\prime}$ étonne: «Pourquoi ils veulent cela ? C' est pour voir à quelle heure ça finit » (Ob 32). Les savoirs ou les connaissances des interlocuteurs sont également remis en cause. On présume et suspecte l' Autre d' être incapable de poursuivre les activités implantées dans le cadre du projet (ong/agents de santé : " $C^{\prime}$ est parce que l' on s' appelle ong que l' on fait cette feuille, et je sais que tu ne le feras pas après » $[\mathrm{V}$ 9]). On croit que les calculs comptables ne sont pas justes (agents de santé/oNG) ou encore que les paysans ne comprennent rien à rien et sont incapables de gérer un centre de santé (ong et/ou agents de santé/ communauté : "Comme c' est la communauté, les gens ils comprennent tout de travers " [Ob 1 $]$ ). Il arrive même quelquefois que ces a priori sous-jacents soient explicitement prononcés par certains acteurs face à d' autres, mais souvent dans une langue que l' Autre n' est pas censé (croit-on) comprendre, le français : «On ne va pas parler avec lui, il comprend rien»(V 11). Dans ce cas, les relations de pouvoir unilatéral entre ces différents protagonistes sont évidemment présentes en permanence et peuvent même parfois déboucher sur un sentiment de condescendance voire de quasi-humiliation. Bourdieu aurait certainement qualifié ces comportements de violence symbolique. 


\section{La substitution}

41 L' avant-dernière logique découverte dans le district sanitaire est celle de la substitution, c' est-à-dire un processus de travail entrepris par des acteurs pour accomplir certaines tâches à la place d' autres personnes.

Ce type de stratégie des acteurs a été rencontré aux deux paliers du système de santé de district, soit dans la relation entre l' ECD et l' ONG et dans celle entre les ICP et les membres des COGES. Dans le premier cas, et à plusieurs reprises, ce dont ne se cache pas le responsable de l' ONG, ses collègues effectuent des tâches ou répondent à des questions à la place des membres de l' ECD. Ils se substituent, en quelque sorte, à l' autorité nationale lorsqu' ils fournissent quelques conseils à la place des responsables nationaux concernant le système de santé. Ce comportement $n^{\prime}$ est pas limité aux seuls membres expatriés de l' ONG, les collaborateurs nationaux (fonctionnaires de l' État) font de même parfois, traduisant l' internalisation de certains comportements. Par exemple, un membre (non habituel) de l' ECD effectuant un contrôle financier avec l' ONG note, sur le cahier du dispensaire pour clore le travail, « inventaire oNG », alors que, dans pareil cas, cette information est habituellement notée par la personne de l' ECD responsable et plus encline à s' approprier le processus «inventaire ONG/ECD » (V 8). Dans le second cas, ce sont certains infirmiers responsables du centre de santé qui agissent ou répondent à la place des membres du COGES. Évidemment, statutairement, ils sont membres de droit des COGES, en tant que secrétaire général, mais ils n' en sont ni le trésorier ni le président. Ainsi, quand ils répondent à la place du président ou qu' ils font des écritures comptables en remplaçant le trésorier, ils se comportent selon une logique de substitution.

Le déploiement $d$ ' une telle stratégie est très certainement la résultante $d$ ' une relation de pouvoir unilatérale entre les deux types $d$ ' acteurs aux deux niveaux du système de santé. Il faut ajouter, contrairement à ce que prétendent certains agents, que cette pratique est connue et comprise des parties prenantes et qu' un processus $d^{\prime}$ accord tacite influence sa persistance. Deux raisons principales paraissent expliquer la présence d' une telle approbation, essentiellement inexprimée par les bénéficiaires de la substitution. La première est la recherche, encore une fois, de la tranquillité, de la paix sociale et de l' absence de conflit (évitement). Remettre explicitement en cause un tel procédé provoquerait des débats et des désaccords que les acteurs ne souhaitent pas voir émerger. La seconde raison est plus pragmatique et s' explique par le fait que les personnes subissant la substitution et celles qui la réalisent peuvent y trouver un intérêt particulier. Les membres de l' ECD peuvent ainsi découvrir bien commode que leurs travaux soient effectués par d' autres, l' ouvrage étant fait. Pour ce qui est des membres de l' ONG, ils s' assurent ainsi de l' avancement de leur projet et de leur capacité à rendre des comptes aux bailleurs de fonds ou aux responsables de l' ong ou du ministère de la Santé. Quant aux infirmiers, outre que cela peut leur permettre, pour les moins honnêtes, $d^{\prime}$ accaparer quelques ressources (accaparement), ils veillent à maintenir leur pouvoir sur les communautés (domination) et à montrer à leur hiérarchie administrative que leur centre de santé fonctionne bien. 


\section{Le mépris du service public}

44 publics et le service public. Elle s' est traduite concrètement par deux types de manifestations. D' un côté, certains agents de santé usent du système public à des fins privées, lorsqu' ils font des consultations privées au sein d' un centre de santé public, par exemple, " $d$ ' autres services empochent 50000 FCFA pour la cataracte, ils empochent » (EI 113) ou lorsqu' un gérant de dépôt de MEG fait payer les pansements auxquels il procède $(\mathrm{Ob} 1)$. $\mathrm{D}^{\prime}$ un autre côté, nous avons rencontré d' autres agents qui ne vont pas se préoccuper $d^{\prime}$ un bien quelconque au seul motif qu' il est de nature publique : «La fonction publique, $c^{\prime}$ est chacun pour soi et Dieu pour tous » (vs 1). On laissera périmer des médicaments donnés par l' État («quand c' est pour l' État!» [vs 4]) ou par des ong à ce dernier. La péremption très prochaine de produits gynécologiques fournis par une oNG $\mathrm{n}$ ' inquiète pas un infirmer: "Comme $c^{\prime}$ est des dotations, $c^{\prime}$ est pas grave » (vs 1).

Deux raisons peuvent expliquer un tel comportement. La première est évidemment liée au fait que la «machine " étatique $\mathrm{n}^{\prime}$ est pas toujours capable de répondre aux demandes et aux besoins de ces agents. Aussi, ils ne souhaitent pas la considérer et vont même parfois jusqu' à vouloir en abuser. La seconde raison est économique, notamment pour les médicaments, car le cas rencontré concerne des dotations gratuites. Or, selon le courant international et national du recouvrement des coûts et de l' importance de disposer d' une source de revenu locale, il n' est pas pertinent de donner des médicaments aux patients, comportement qui est d' autant plus aisé que les produits proviennent directement de l' État.

\section{La rente du développement et les logiques d'acteurs}

La mise en lumière de ces différentes logiques dans le contexte particulier de la recherche, outre l' apport modeste au développement des connaissances socioanthropologiques, vise à rendre compte de la complexité des rapports sociaux associés à l' implantation d' un projet de développement lié à une politique de santé. Il s' agit de tenter, par la présentation de données vivantes et vécues, de montrer que les explications simplistes ne peuvent plus avoir court. La dichotomie entre les acteurs du Nord et du Sud - employée par certains pour comprendre les échecs des projets, des programmes ou des politiques de santé publique - ne nous paraît plus, heuristiquement parlant, d' intérêt. Cette grille de lecture opposant le Nord et le Sud est également dénoncée par Piot (directeur de l' ONUSIDA) et Caraël (Piot \& Caraël 2005) dans un ouvrage récent consacré à $\mathrm{l}^{\prime}$ épidémie du sida et à la théorie du complot. Navarro (2004) demande que la vision de la domination du Nord par le Sud soit écartée au profit d' une compréhension d' un monde dominé par des alliances sociales tant au Nord qu' au Sud, et ce, pour contrer les velléités de redistribution des ressources au détriment de ces alliances - nous ne pouvons ici montrer la pertinence de son propos lorsqu' il s' agit d' évoquer le concept de pouvoir (Ridde 2007). Dans le cas étudié par la présente recherche, il nous semble que l' explicitation de ces logiques $d^{\prime}$ acteurs contribue, en partie, à comprendre pourquoi les acteurs ont essentiellement souhaité orienter leurs efforts, lors de la mise en œuvre de l' Initiative de Bamako, vers l' atteinte des objectifs relatifs à l' efficacité au détriment de ceux liés à l' équité. Pour le 
dire autrement et confirmant empiriquement certaines propositions théoriques (Flori \& Tizio 2000), les acteurs sociaux, qu' ils soient membres de l' ONG, des COGES ou encore agents du système de santé, usent de l' équation «efficacité ergo équité », ce dont la logique de l' évitement rend parfaitement compte. Cela a été montré dans la recherche dont il est ici question (Ridde 2008), comme d' autres l' ont fait avant nous ailleurs en Afrique (Gilson 2000). Dans un hôpital pédiatrique du Burkina, cette logique de l' évitement a déjà été mise au jour dans le cas de la prise en charge des enfants victimes $d u$ sida. Les infirmières se retrouvent obligées $d$ ' adopter une telle stratégie pour éviter de révéler « au grand jour la double impuissance du soignant et du parent » (Desclaux 1999 : 545), les premiers ne pouvant proposer de traitements efficaces et les seconds acheter ce que les premiers ont malgré tout prescrit.

Les éléments précédemment mentionnés concernant les logiques d' acteurs favorisent donc beaucoup plus l' immobilisme et le statu quo que le changement social, système et individus participant à l' explication. Les agents de santé ont "capturé » le système, nous disent l' ancien responsable de l' IB à l' Unicef (Paganini 2004) et un expert africain de l' oms (Nyamwaya 2003). Mais ils sont aussi responsables d' un système où chacun neutralise l' autre (logiques de neutralisation) dans une organisation fort complexe, très hiérarchique. Jaffré (1999) explique parfaitement comment les jeunes sagesfemmes qui sortent de l' école sont scandalisées par certaines pratiques professionnelles. Mais, après quelques années de routine au sein du système, elles adoptent finalement les pratiques qu' elles critiquaient, ce qui traduit l' incapacité de lutter contre ce système de contraintes. Toujours au plan du système, si au Burkina certains agents - contraints par la logique systémique du "recouvrement des coûts » et de la privatisation du financement de la santé - retiennent les cartes d' identité des patients ne pouvant pas payer, la situation peut être paroxystique ailleurs. Au Burundi, comme semble-t-il dans d' autres pays en Afrique centrale, ce ne sont pas les cartes mais les patients eux-mêmes qui sont retenus dans les hôpitaux. Surveillés par des gardiens du secteur privé, ils ne peuvent sortir de l' enceinte avant d' avoir payé, certains restant ainsi détenus plusieurs semaines (Kippenberg et al. 2008). Au niveau des individus, nous avons remarqué, en outre, que pour certains agents et membres de comités de gestion, la logique de l' accaparement de la rente du développement est largement mobilisée. Ceci explique en partie, comme d' autres l' ont dit sur l' IB (Tizio \& Flori 1997), le fait que ces personnes sont plus attirées par la maximisation de leurs profits que par la redistribution au profit des indigents : « Ils sont plus préoccupés par leurs salaires » (EF 18), nous dit un paysan. La formation est la source de revenus supplémentaires la plus citée par des médecins africains (Roenen et al. 1997). L' impact de la pratique des per diem mise en évidence dans la présente recherche, comme dans quelques très rares autres études au Mozambique (Pfeiffer 2003), au Nigeria (Smith 2003) ou au Mali (Berche 1998), est relativement néfaste pour l' organisation du système de santé (Ridde 2010). Et il faut comprendre ici que c' est la présence des acteurs du Nord et le fonctionnement par projets (qu' ils soient gérés par des Burkinabè ou des expatriés) ainsi que la dépendance à l' égard de ces derniers qui induisent de telles pratiques. Le per diem est aujourd' hui devenu quasiment un droit et « seem to top all other extra income activities regarding frequency and effectiveness " (Roenen et al. 1997). Il devient maintenant impossible de faire quoique ce soit sans ces per diem. La concurrence entre les projets (Pfeiffer 2004) et la faiblesse des salaires des fonctionnaires (van Lerberghe et al. 2002) face à un coût de la vie toujours croissant ont 
participé à la généralisation de la pratique et des logiques subséquentes. Comme nous l' avons vu pour les sages-femmes, ces pratiques frauduleuses sont également dénoncées par beaucoup, mais les pourfendeurs ne parviennent pas à lutter véritablement contre celles-ci, tant le système est « enchâssé dans le système administratif que toute réforme est particulièrement difficile, et que les réformateurs sont marginalisés " (Blundo \& Olivier de Sardan 2001: 10). Globalement, nous dit van Balen (2004) concernant les soins de santé primaires, "We also underestimated the conservatism of the medical establishment and the time the appropriation of the change by the actors involved requires in diverse circumstances » (p. A25).

Ces logiques d' acteurs sont utiles pour comprendre pourquoi aucun entrepreneur politique $\mathrm{n}^{\prime}$ est apparu dans l' arène de la recherche pour faire en sorte que l' on se préoccupe de la question de l' équité. À partir du moment où des stratégies d' évitement, de connivence ou de mépris du service public s' opèrent, personne ne souhaite user de ses ressources et mettre tout en œuvre pour que la situation que vivent les indigents devienne un problème public auquel une solution doit être apportée (Ridde 2006b). Pour que des personnes soient qualifiées d' entrepreneurs, nous dit Kingdon (1995), elles doivent disposer de trois qualités principales. Elles doivent être persistantes, savoir négocier et être écoutées et reconnues grâce à leur expertise, leur leadership ou leur position stratégique de décideur. Elles doivent aussi être toujours prêtes à agir pour mettre leurs idées sur le devant de la scène au moment opportun. Les résultats, en partie exposés supra, montrent clairement que de tels personnages $\mathrm{n}^{\prime}$ ont pas existé au sein de $\mathrm{l}^{\prime}$ arène de la présente recherche lors du processus de mise en œuvre de l' IB. Sans pour autant tomber dans un déterminisme à tout crin, cette étude tend à montrer que dans ce système de contraintes particulier, le potentiel créatif des individus, pour reprendre les mots de Strauss, ne s' est pas fait ressentir au profit des plus pauvres. Ce potentiel existe, et a certes été mobilisé, mais plutôt en faveur de ceux disposant déjà d' une position favorable dans le système social ou le système de santé.

La teneur de notre discussion suite à la présentation des données empiriques, pourrait faire croire à un halo de pessimisme permanent. Or, ce n' est point le cas puisque deux personnes ont fait mine $d^{\prime}$ user de leurs ressources pour instaurer une plus grande justice sociale dans le système de santé burkinabé. Cela n' a pas été le cas dans le district étudié, mais l' a été dans l' hôpital régional vers lequel les agents de santé sont censés envoyer leurs patients victimes de complications (Ridde 2007). Cependant, dans le contexte précis de la recherche, l' absence d' entrepreneur mobilisé en faveur de l' équité $d$ ' accès aux soins a été observée et s' explique en partie par la mobilisation des logiques d' acteurs décrites préalablement.

Si l' éthique des rapports Nord/Sud constitue une clé pour la compréhension des échecs de certaines politiques publiques de santé, entre autres au regard de leur volonté d' instaurer plus d' équité et de justice sociale, cet article démontre que $d^{\prime}$ autres éléments contribuent à rendre intelligibles ces revers. Les relations de pouvoir et de domination entre les acteurs sociaux, quel que soit leur positionnement stratégique ou géographique, forment une autre clé utile pour élucider les difficultés d' émergence et de mise en œuvre du changement social. Les acteurs humanitaires du Nord, tout comme les médecins ou les sages-femmes du Sud, ne sont pas, ipso facto, préoccupés par l' équité ou la justice sociale. Nous pourrions convoquer la fameuse «inverse equity 
hypothesis » (Victora et al. 2000) qui propose que les nouvelles interventions de santé publique ne profitent pas immédiatement aux plus pauvres mais $\mathrm{d}^{\prime}$ abord aux plus riches. Sans évoquer les pamphlets romancés récents, les critiques de l' aide humanitaire et de ses acteurs ne manquent pas (Hours 1998 ; Sogge 2003). Certains sont allés jusqu' à interroger le lien entre les ONG, l' aide humanitaire et la guerre (Pérouse de Montclos 2002). Nous n' irons pas jusque-là. Cependant, l' étude fait valoir que, dans l' analyse de l' interprétation et de la mise en œuvre des réformes sanitaires par les acteurs sociaux, en particulier les réformes imposées sinon incitées par le Nord, le concept de pouvoir doit être pris en compte (Collins et al. 1999). Effectivement, «c' est par l' exercice du pouvoir que se réalisent ou non les politiques publiques, bien loin de se dérouler d' elles-mêmes" (Lemieux 2002 : 22). L' étude de l' interaction entre les acteurs que propose l' approche de l' anthropologie du développement employée dans cet article mérite également d' être complétée, pour des raisons heuristiques, par l' analyse de la question du pouvoir et du contrôle des ressources par les acteurs sociaux. Aussi, nous émettons l' hypothèse que le pouvoir doit être compris comme la source et la cause sous-jacente de l' émergence de ces logiques d' acteurs mises au jour dans ce texte. Il nous reste donc à comprendre sur quelles ressources portent majoritairement l' exercice du pouvoir en fonction des différentes logiques rencontrées et à vérifier si ces ressources en jeu forment des atouts ou des enjeux, pour reprendre la dichotomie de Crozier et Friedberg (1977).

\section{BIBLIOGRAPHIE}

ABSP 2001 Rapport de l' atelier de discussion des résultats du projet MAPHEALTH, Ouagadougou, Association burkinabé de santé publique : 6 .

BADINI, A. 1994 Naître et grandir chez les Moosé traditionnels, Paris, Sépia (« Découvertes du Burkina »); Ouagadougou, ADDB.

VAN BALEN, H. 2004 " Disease Control in Primary Health Care : A Historical Perspective ", Trop Med Int Health, $6:$ A22-A26.

Balique, H., Ouattara, O. \& Ag Iknane, A. 2001 " Dix ans d' expérience des centres de santé communautaire au Mali », Santé publique, $13: 35-48$.

BERCHE, T. 1998 Anthropologie et santé publique en pays dogon, Paris, Karthala.

BEYER, B. 1998 " The Politics of the Health Care District Reform in The Republic of Benin ", International Journal of Health Planning and Management, $13: 230-243$.

Blundo, G. \& Olivier DE SARDAN, J.-P. 2001 " La corruption quotidienne en Afrique de l' Ouest », Politique africaine, $83:$ 8-36.

BRINKERHOFF, D. W. 1996 " Process Perspectives on Policy Change : Highlighting Implementation », World Development, 24 : 1395-1401. 
BRUGHA, R. \& VARVASOVSZKY, Z. 2000 " Stakeholder Analysis : A Review ", Health Policy and Planning, $15: 239-246$

BRUNET-JAILLY, J. 1992 " Santé : une occasion manquée ? Le Mali et l' Initiative de Bamako ", Afrique contemporaine, $162: 3-18$.

ColLINS, C. 1994 Management and Organization of Developing Health Systems, Oxford, Oxford University Press.

Coluins, C., Green, A. \& HunTeR, D. 1999 " Health Sector Reform and the Interpretation of Policy

Context ", Health Policy, $47:$ 69-83.

CROzIER, M. \& FRIEDBERG, E. 1977 L' acteur et le système, Paris, Éditions du Seuil.

Deschamps, J.-P. 2000 " Les enjeux de santé publique dans le pays en voie de développement », Médecine \& Sciences, 16 : 1211-1217.

DesclauX, A. 1999 " Des infirmières face au sida. Impact de l' épidémie sur les rôles professionnels dans un service de pédiatrie du Burkina Faso ", in C. BECKER, J.-P. DOzon, C. OBBo \& M. TOURÉ (dir.), Vivre et penser le sida en Afrique, Paris, Karthala : 541-558.

EMERSON, R. M., FRETZ, R. I. \& SHAW, L. L. 1995 Writing Ethnographic Fieldnotes, Chicago-London, University of Chicago Press.

FLORI, Y.-A. \& TiZIO, S. 2000 " Les politiques sanitaires subsahariennes : efficacité versus équité ou efficacité ergo équité », in A. Alcouffe, B. Fourcade, J.-M. Plassard \& G. TAHAR (dir.), Efficacité versus équité en économie sociale, Paris, L' Harmattan : 467-478.

GILSON, L. 1997 Implementing and Evaluating Health Reform Processes : Lessons from the Literature, Bethesda, PHR-Abt Associates.

2000 " Readdressing Equity : The Importance of Ethical Processes ", in A. MiLls (ed.), Reforming Health Sectors, London-New York, Kegan Paul International : 103-122.

Gilson, L., Kalyalya, D., Kuchler, F., Lake, S., ORgana, H. \& Ouendo, M. 2000 ” The Equity Impacts of Community Financing Activities in Three African Countries ", International Journal of Health Planning and Management, 15 : 291-317.

GRIndLe, M. S. \& Thomas, J. W. 1991 Public Choices and Policy Change. The Political Economy of Reform in Developing Countries, Baltimore-London, The Johns Hopkins University Press.

HouRs, B. 1998 L'idéologie humanitaire ou le spectacle de l' altérité perdue, Paris, L' Harmattan. 2001 Systèmes et politiques de santé : de la santé publique à l' anthropologie, Paris, Karthala.

JAFFRÉ, Y. 1999 " Les services de santé “pour de vrai”. Politiques sanitaires et interactions quotidiennes dans quelques centres de santé (Bamako, Dakar, Niamey) », Bulletin de l' APAD, 17 : 3-17.

JAFFRÉ, Y. \& OLIVIER DE SARDAN, J.-P. (dir.) 2003 Une médecine inhospitalière. Les difficiles relations entre soignants et soignés dans cinq capitales d' Afrique de l' Ouest, Paris, Karthala (« Hommes et sociétés »). Kingdon, J. W. 1995 Agendas, Alternatives and Publics Policies, New York, Harper Collins.

Kippenberg, J., SAHokwasama, J. B \& Amon, J. J. 2008 " Detention of Insolvent Patients in Burundian Hospitals », Health Policy Plan, 23 : 14-23. 
Knippenberg, R., Alihonou, E., Soucat, A., Ndiaye, J.-M., LAMARque, J.-P. \& el Abassi, A. 1997 " L' enfant en milieu tropical », in R. KNIPPENBERG ET AL. (dir.), Huit ans d' expérience de l' Initiative de Bamako, Paris, CIE : 1-108.

LAsCoumes, P. \& Le Galès, P. 2007 Sociologie de l' action publique, Paris, Armand Collin.

LAURent, P.-J. 1996 " Dynamiques matrimoniales chez les Mossi du Burkina Faso. Pratiques de l' église des Assemblées de Dieu, des aînés, des cadets et de l' État », in G. DE VILLIERS (dir.),

Phénomènes informels et dynamiques culturelles en Afrique, Bruxelles, Institut africain-CEDAF ; Paris, L' Harmattan : 166-183.

Lemieux, V. 2002 L'étude des politiques publiques, les acteurs et leur pouvoir, Québec, Les Presses de l' Université Laval.

VAn Lerberghe, W., Conceicao, C., VAn Damme, W. \& Ferrinho, P. 2002 " When Staff is Underpaid : Dealing with the Individual Coping Strategies of Health Personnel », Bull World Health Organ, 80 : 581-584.

LoNG, N. \& LoNG, A. (eds.) 1992 Battlefields of Knowledge. The Interlocking of Theory and Practice in Social Research and Development, London, Routledge.

MaÏGA, Z., TRAORÉ NAFO, F. \& El ABASSI, A. 1999 La réforme du secteur santé au Mali 1989-1996, Antwerp, Belgium, ITG Press («Studies in health services organisation \& policy »).

MEUWISSEN, L. E. 2002 " Problems of Cost Recovery Implementation in District Health Care : A Case Study from Niger », Health Policy Plan, 17 : 304-313.

MinISTÈRE DE LA SANTÉ 1994 " Note introductive sur l' Initiative de Bamako au Burkina Faso », Bilan des années 1992 et 1993 et perspectives dans le contexte de la dévaluation du CFA présent à l'occasion de la Rencontre nationale avec les partenaires de l' initiative de Bamako, Ouagadougou, Secrétariat général : 13.

NAVARRO, V. 2004 " The World Health Situation », International Journal of Health Services, 34 : 1-10.

NitiÈMA, A., RidDE, V. \& GIRARD, J.-E. 2003 " L' efficacité des politiques publiques de santé dans un pays de l' Afrique de l' Ouest : le cas du Burkina Faso ", International Political Science Review, 24 : 237-256.

Nougtara, A., Haddad, S., OUÉdraogo, J. G., KY-ouÉdRAogo, S., Ridde, V. \& Fournier, P. 2007 " Health Sector Reform under Macroeconomic Adjustment in Burkina Faso : Lost Opportunities ? ", in S. HADDAD, E. BARIS \& D. NARAYANA (eds.), Safeguarding the Health System in Times of Macroeconomic Instability. Policy Lessons for Low and Middle-Income Countries, Trenton, Africa World Press-IDRC : 87-130.

NYAmWAYA, D. 2003 " Health Promotion in Africa : Strategies, Players, Challenges and Prospects ", Health Promot Int, 18 : 85-87.

OLIVIER DE SARDAN, J.-P. 1990 " Sociétés et développement », in D. FASSIN \& Y. JAFFRÉ (dir.), Sociétés, développement et santé, Paris, Ellipses : 28-37.

1995 Anthropologie et développement. Essai en socio-anthropologie du changement social, Paris, Karthala.

2000 " Dramatique déliquescence des États en Afrique ", Le Monde Diplomatique, février : 12-13.

2002 " Les trois approches en anthropologie du développement ", Revue Tiers-Monde, 168 : 737-762. 
2007 " De la nouvelle anthropologie du développement à la socio-anthropologie des espaces publics africains », Revue Tiers-Monde, $191: 543-552$.

OUÉDRAOGO, J. B. 1996 " The Articulation of the Moose Traditional Chieftaincies, the Modern Political System, and the Economic Development of Kaya Region », Journal of Legal Pluralism, 37-38 : 249-261.

Ouendo, M., Makoutode, M., Agueh, V. \& Manko D' Almeida, A. 2000 Équité dans l' application de l' Initiative de Bamako : situation de la prise en charge sanitaire des indigents au Bénin et approche de solution, Actes de la conférence internationale « Financement des systèmes de santé dans les pays à faible revenu d' Afrique et d' Asie ", décembre, Clermont-Ferrand.

Paganini, A. 2004 " The Bamako Initiative Was not about Money ", Health Policy and Development, $2: 11-13$.

Palmer, N., Mueller, D. H., Gilson, L., Mills, A. \& Haines, A. 2004 " Health Financing to Promote Access in Low Income Setting : How Much do We Know ?», The Lancet, 364 : 1365-1370.

PÉRouse de Montclos, M.-A. 2002 L' aide humanitaire, aide à la guerre ?, Bruxelles, Complexe.

PfEIFfER, J. 2003 " International NGOs and Primary Health Care in Mozambique : The Need for a New Model of Collaboration », Social Science and Medicine, 56 : 725-738.

2004 " International NGOs in the Mozambique Health Sector: The "Vevet Glove" of Privatization », in A. CASTRO \& M. SINGER (eds.), Unhealthy Health Policy. A Critical Anthropological Examination, Walnut Creek, Altamira Press : 43-62.

PIOT, P. \& CARAËL, M. 2005 L' épidémie de sida et la mondialisation des risques, Loverdal, Labor.

RATHWELL, T. 1998 " Implementing Health Care Reform : A Review of Current Experience ", in R. B. SALtMAn, J. Figueras \& C. SAKellarides (eds.), Critical Challenges for Health Care Reform in Europe, Buckingham Philadelphia, Open University Press : 385-399.

RIDDE, V. 2003 " Fees-for-Services, Cost Recovery, and Equity in a District of Burkina Faso Operating the Bamako Initiative », Bulletin of World Health Organization, 81 : 532-538.

2006a « Appréhender les conceptions locales de l' équité pour formuler les politiques publiques de santé au Burkina Faso », Promotion \& Éducation, XIII : 252-256.

$2006 \mathrm{~b}$ « L' accès des indigents aux services de santé au Burkina Faso : un problème public ? ", Lien social et Politiques, 55 : 149-163.

2007 Équité et mise en œuvre des politiques de santé au Burkina Faso, Paris, L' Harmattan.

2008 " "The Problem of the Worst-off is Dealt with After All Other Issues" : The Equity and Health Policy Implementation Gap in Burkina Faso », Social Science and Medicine, 66 : 1368-1378.

2010 " Per diems Undermine Health Interventions, Systems and Research in Africa : Burying Our Heads in the Sand ", Tropical Medicine and International Health, 28, In press.

RIDDE, V. \& GIRARD, J.-E. 2004 " Douze ans après l' initiative de Bamako : constats et implications politiques pour l' équité $d$ ' accès aux services de santé pour les indigents africains ", Santé publique, $15: 37-51$.

Roenen, C., Ferrinho, P., VAn Dormael, M., Conceiçaõ, M. C. \& VAn Lerberghe, W. 1997 " How African Doctors Make Ends Meet : An Exploration », Tropical Medicine \& International Health, 2 : 127-135. SMITH, D. J. 2003 " Patronage, per diems and the "Workshop Mentality" : The Practive of Family Planning Programs in Southeastern Nigeria », World Development, 31 : 703-715. 
SOGGE, D. 2003 Les mirages de l' aide internationale. Quand le calcul l' emporte sur la solidarité, Paris, Enjeux Planète.

STAKE, R. E. 2000 " Case Studies ", in N. K. DENZIN \& Y. S. LinColn (eds.), Handbook of Qualitative Research, London, Sage Publications : 435-454.

STRAUSS, A.-L. 1992 La trame de la négociation : sociologie qualitative et interactionnisme, Paris, L' Harmattan.

TIZIO, S. \& FloRI, Y.-A. 1997 " L' initiative de Bamako : "santé pour tous" ou "maladie pour chacun"?», Revue Tiers-Monde, XXXVIII : 837-858.

Victora, C. G., Vaughan, J. P., Barros, F. C., Silva, A. C. \& Tomasi, E. 2000 " Explaining Trends in Inequities : Evidence from Brazilian Child Health Studies », Lancet, 356 : 1093-1098.

WALT, G. 1994 Health Policy: An Introduction to Process and Power, London, Zed Press ; Johannesburg, University of Witwaterstand.

WALT, G. \& GILSON, L. 1994 " Reforming the Health Sector in Developing Countries. The Central Role of Policy Analysis », Health Policy and Planning, 9 : 353-370.

WINTER, G. (dir.) 2001 " Inégalités et politiques publiques en Afrique : pluralités des normes et jeux d' acteurs ", Paris, Karthala, IRD.

YIN, R. K. 1994 Case Study Research Design and Method, London-New Delhi, Sage Publications («Applied Social Research Methods Series, 5 »).

\section{NOTES}

1. Les experts dans l' étude des politiques publiques font aussi parfois appel à ce concept (LASCOUMES \& LE GALÈS 2007).

2. Je tiens à remercier tous les collègues du ministère de la Santé, à Ouagadougou et en périphérie, qui ont su se rendre disponibles pour répondre à mes multiples questions, me trouver les bons documents et me diriger vers les bons interlocuteurs. De même, l' ONG et tous ses membres $\mathrm{m}$ ' ont apporté un secours considérable et une confiance importante. La mise à disposition de certains de leurs moyens et les discussions que nous avons entreprises ensemble $\mathrm{m}^{\prime}$ ont permis $\mathrm{d}^{\prime}$ accomplir cette recherche. $\mathrm{Qu}$ ' ils soient tous ici remerciés ainsi que Touendé Bertrand pour son aide à la collecte des données et à leur traduction. Cette collecte a été financée par le Centre de recherche en développement international (CRDI) du Canada. Je détiens une subvention de chercheur (New Investigator) des Instituts de recherche en science de la santé (IRSC) du Canada.

3. Au moment où $s^{\prime}$ est déroulée cette recherche, le Burkina Faso disposait de 13 régions sanitaires correspondant aux 13 régions administratives et 55 districts sanitaires qui, à l' exception des villes de Ouagadougou et de Bobo Dioulasso, correspondent aux limites des entités provinciales.

4. Il s' agit des codes employés dans l' ouvrage dont est issu cet article pour retrouver les auteurs des verbatim, EF : entrevue formelle; EI: entrevue informelle; $\mathrm{Ob}$ : observation ; V : visite ; FG : focus group ; VS : visite de supervision ; voir RIDDE (2007). 


\section{RÉSUMÉS}

RÉSUMÉ

Les échecs relatifs des politiques de santé favorables à l' équité en Afrique sont souvent expliqués par le prisme de la déficience de leur contenu et des modalités de mise en œuvre au détriment d' une compréhension fine du jeu des acteurs dans ce processus. Or, l' analyse de tels échecs ne peut faire l' économie de l' étude des logiques de comportements des acteurs sociaux. Aussi, pour ce faire, une recherche est menée dans un district sanitaire du Burkina Faso à l' aide d' une enquête de terrain de type socio-anthropologique. Cet article documente empiriquement la récurrence de logiques particulières qui permet de mieux comprendre ces échecs relatifs. Les logiques mises au jour sont l' accaparement, la neutra/domination, le discours, le clientélisme, l' opacité, la connivence, l' évitement, la suspicion, la substitution, le mépris du service public. L' étude fait valoir que dans l' analyse de l' interprétation et de la mise en œuvre des réformes sanitaires par les acteurs sociaux le concept de pouvoir doit être pris en compte.

\section{ABSTRACT}

Public Policies on Health, Actor's Logics and Negociated Order in Burkina Faso.

The relative failures of equity-promoting health policies in Africa are often attributed to shortcomings of content or implementation modalities, thereby sidestepping any in-depth understanding of the actors' roles in this process. Yet any analysis of such failures cannot afford to neglect the social actors' behaviour logic. Therefore, to this end, we conducted a study in a health district of Burkina Faso using a socio-anthropological field survey. This article empirically documents the recurrence of specific logics that provide a better understanding of these relative failures. The logics uncovered were: monopolizing, neutralization/domination, discourse construction, clientelism, opacity, connivance, avoidance, suspicion, substitution and disdain for public service. The study emphasizes the need, when analyzing the interpretation and implementation of health reforms by social actors, to take into account the concept of power.

\section{INDEX}

Keywords : Burkina Faso, Clientélisme, équité, Mise En œuvre, Logiques, Politiques Publiques, Pouvoir, Santé

\section{AUTEUR}

\section{VALÉRY RIDDE}

Département de médecine sociale et préventive, Centre de recherche du Centre hospitalier de l' Université de Montréal, Canada ; Institut de recherche en sciences de la santé du CNRST, Ouagadougou, Burkina Faso. 